\title{
Electronic transmission of nonlocal suppressive effect of Chinese herbal medicine to Escherichia coli
}

\author{
Yu Chen, Zhong Zhen Cai, Peng Gao, Qian Feng, Xuemei Bai* and Bruce QTang* \\ Institute of Life Science and Technology, ENN group, Langfang, China
}

\begin{abstract}
A number of nonlocal biological effect of chemicals were reported in recent years. In this study, we examined nonlocal effect of two antibacterial Chinese medicinal herbs (Houttuynia cordata Thunb and Sargentodoxa cuneata) on the growth of Escherichia coli. The processed herbs were placed on the transmission side of a commercially available scalar wave device, and the E. coli cultured on triplicate agar plates on the receiver side. Growth suppression was evaluated by Colony Forming Unit (CFU) determination in comparison to the control group. A significant nonlocal inhibitory effect (up to $30 \%$ ) was demonstrated for the treatment group (p<0.05), indicating a success in transmitting the antibacterial function of the herbs to the $E$. coli cultures. Factors affecting the success rate were explored.
\end{abstract}

\section{Introduction}

Energy medicine provides a new medical choice for patients and holds great promise for its non-invasive nature [1]. From the perspective of physics, all lives depend upon the molecular interacting via vibrating energy fields as molecules, atoms, electrons, and chemical bonds all have their vibration characteristics [2]. For the past twenty years, many studies have demonstrated the feasibility of nonlocal effects on the viabilities of microorganism via electronic transmission of the information from the targeting molecules [3-6]. Compared with non-thermal inactivation technologies through the direct effect of electromagnetic field [7], the electronic transmission of nonlocal effect depends on the signals of the special chemical drugs [3,6].

To date, most of the effective experimental results were based on the relative simple chemical molecule and antibiotics, such as: acetic acid [8], phorbol-myristate acetate [9], retinoic acid [3], ampicillin [5], vancomycin [4], penicillin [1] and clotrimazole [6]. Some of the electronic information delivery was mediated through aqueous system $[1,4,5]$ which is believed to be able to generate dissipative nanostructures when induced by exogenous physical fields, providing basis for storing and retrieving biologic information [3]. Other studies showed that the electronic transmission did not depend upon the aqueous system; instead, it utilized special antennas from both the molecules on the transmission side and the biological organisms on the receiver side $[6,10,11]$.

There is only very limited amount of work conducted in this area of nonlocal effect so far even though it's a very important phenomenon to explore. More scientific evidence is needed to make advance in this field, and the attempt to explain the mechanism of this effect will allow us to better understand the correlation of matter, information and energy.

In this study, we used two antibacterial Chinese herbal medicines as source of signals to evaluate the electronic transmission of nonlocal effects of more complex and non-artificial synthetic substance. Houttuynia cordata Thunb and Sargentodoxa cuneata were chosen, as both have well-documented antibacterial effects $[12,13]$. For the signal transmission, a commercially available device was used, which was reported to be able to transfer specific molecular signals onto target cells through scalar waves $[6,10]$. Furthermore, the different physical properties (liquid or solid) of the source material were compared to understand whether the electronic transmission of nonlocal effects was aqueous system dependent.

\section{Materials and methods}

\section{Chinese herb material and preparation of extracts}

The Chinese herbal medicine of Houttuynia cordata Thunb and Sargentodoxa cuneata were purchased at Chinese medicine store (Tongrentang chain-store). The dried H. cordata (25 g) and S. cuneata $(25 \mathrm{~g})$ were extracted with boiling water for $2 \mathrm{~h}$ and filtered through a $0.45 \mu \mathrm{m}$ filter (Osmonics, Minnetonka, MN, USA), followed by freezedrying yielding a fine powder as the final product to be stored at $4^{\circ} \mathrm{C}$.

The direct inhibitory effect was tested by introducing different concentrations of the medicine in the range of $125-10000 \mu \mathrm{g} / \mathrm{mL}$ to the culture medium, followed by cell count determination after incubation for $1 \mathrm{~h}$ at $37^{\circ} \mathrm{C}$. The concentration that can achieve $100 \%$ inhibition of growth was used for the subsequent nonlocal experiments.

The nonlocal inhibitory effect was examined by using two sources, with the first one being a $5 \mathrm{ml}$ of diluted solution of the processed herbs at the concentration of $1250 \mu \mathrm{g} / \mathrm{mL}$ (Source A), and the second one $1 \mathrm{~g}$ of dried power (Source B).

${ }^{*}$ Correspondence to: Xuemei Bai, Institute of Life Science and Technology, ENN group, Langfang, 065001, China, E-mail: baixuemei@enn.cn

Bruce Q Tang, Institute of Life Science and Technology, ENN group, Langfang, 065001, China, E-mail: tangqing@enn.cn

Key words: Chinese medicinal herb, nonlocal biological effect, electronic transmission, Escherichia coli

Received: February 01, 2019; Accepted: February 11, 2019; Published: February 14,2019 


\section{Bacteria and preparation}

E. coli strain DH5a (obtained from the Institute of ENNOVA Life Science and Technology) was used for this study. The strain was maintained in Luria-Bertani $(L B)$ medium $\left(1.5 \%\right.$ agar) at $4^{\circ} \mathrm{C}$ in the freezer. For each set of experiment, it was taken from freezer and expanded in a $50 \mathrm{ml}$ Erlenmeyer flask containing $30 \mathrm{ml}$ of $L B$ liquid medium (yeast extract $5 \mathrm{~g} / \mathrm{L}$, peptone $10 \mathrm{~g} / \mathrm{L}, \mathrm{NaCl} 10 \mathrm{~g} / \mathrm{L}, \mathrm{pH} 7.0$ ) by placing on a rotary shaking incubator set at $200 \mathrm{rpm}$ and $37^{\circ} \mathrm{C}$ for $12 \mathrm{~h}$. This process ensured that bacteria were activated into the exponential growth stage to be used as inoculum for subsequent experiments.

\section{Electronic transmission of Chinese herbal medicine property}

Scalar wave experimental kit was purchased from Germany. As shown in Figure 1, this device consists of two capacitor plates (flat Tesla coils) with ball electrodes connected by a cable, one working as transmitter and the other receiver. The transmitter is run by a frequency generator which can be modulated in amplitude. At a particular frequency, self-resonance between the transmitter and receiver can be achieved. In our experiments, the resonant frequency varied between 4.3 and $4.7 \mathrm{MHz}$, and more detailed descriptions were provided by Myle et al. [6].

\section{Evaluation of suppressive effect of electronic transmission signals}

During the exposure experiments, the Chinese herb extract (source A or source B) was placed on the transmitter and the biological target (bacterial plates) on the receiver to assess the nonlocal biological effects. The exposure time was $4 \mathrm{~h}$ and $48 \mathrm{~h}$ respectively, and the temperature was set at $31^{\circ} \mathrm{C}$.

To prepare the bacterial plates, the activated cells of E. coli were spread on $L B$ agar medium in plastic petri dishes at a density of 150 200 cells/dish. Cell viability was determined by counting the colony forming units (CFU) where the suppressive effect was evaluated as a percentage of the control group, which were set up under the same condition but with no irradiation from the device. Blanks were samples placed on the receiver side of the device with no medicine on the transmission side, which was used to evaluate the effect of the device alone. Each exposure experiment was independently repeated for three times. A one-way ANOVA test was performed for the data analysis.

\section{Reagents used}

Peptone, yeast extract, agar (Sigma, USA), and other reagents of analytical grade (Tianjin Fengchuan Chemical Reagent Co., Ltd., China) were used.

\section{Results}

\section{Direct antibacterial effect of $\boldsymbol{H}$. cordata and S. cuneata}

As shown in Table 1 , the extract of $H$. cordata and S. cuneata can inhibit the growth of $E$. coli effectively, with the inhibition rate increasing with the concentration of the medicine. At the concentration of $1250 \mathrm{ug} / \mathrm{mL}$, a nearly $100 \%$ inhibitory effect was obtained.

\section{Nonlocal antibacterial effect of $H$. cordata and S. cuneata}

As shown in Figure 2, a nonlocal inhibitory effect of $H$. cordata and S. cuneata was achieved for source B, with cell viability decreased by $27 \%$ $(\mathrm{p}<0.01)$ compared to the non-irradiated control group. However, there was no significant change $(\mathrm{P}>0.05)$ observed for the group exposed to the information of source A, despite the fact that a near $100 \%$ of growth inhibition was observed under the direct chemical influence of source A (Table 1). This result showed that nonlocal suppressive effect of electro-transferred medicine was depended on the physical properties of the information source. No significant difference between the blank group and the control group was observed, indicating receiving the electronic transmission alone could not inhibit bacterial growth.

Compared to the direct contact of the medicine, the nonlocal antibacterial function takes much longer time to take effect. As shown in Figure 3, no significant inhibition was observed for exposure to the source B signal for $4 \mathrm{~h}$, and only under the long exposure time with electro-transferred Chinese herb signal, a significant nonlocal suppressive effect (up to 27\%) was obtained. Without the coupling of the electromagnetic signal of the medicine, the "blank" electronic transmission field did not affect the cell growth regardless of the exposure time. Therefore, the electronic transmission of nonlocal suppressive effects, unlike regular direct chemical antibacterial effect, requires an accumulation process.

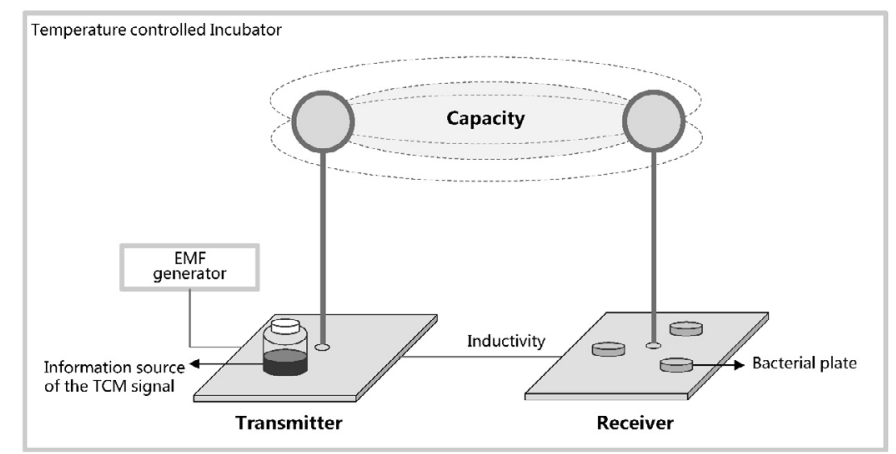

Figure 1. Schematic representation of the electronic transmission setup

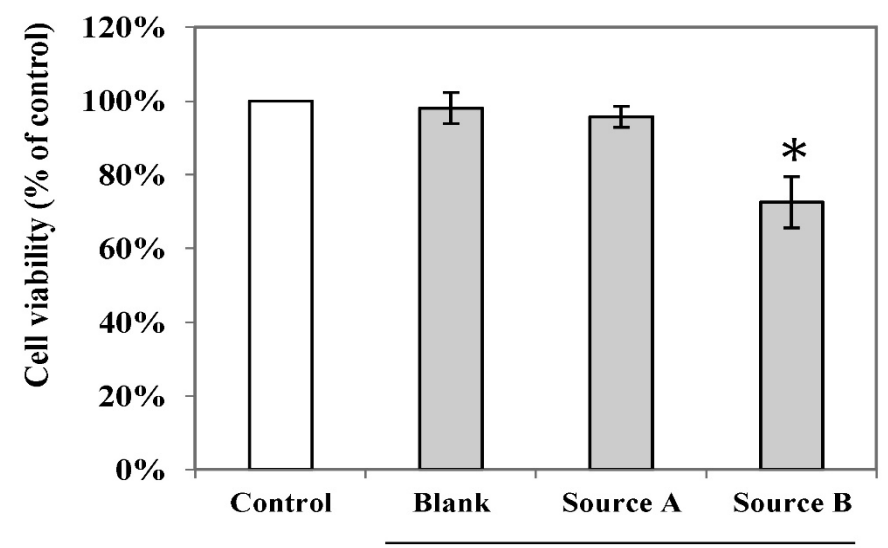

Information source

Figure 2. Cell viability of $E$. coli exposed to the electronic transmission of different information source. Values reported as percentages of the control group. Star indicating a value significantly different from the control group $(* \mathrm{p}<0.01)$

Table 1. Direct inhibitory effect of different concentrations of $H$. cordata and $S$. cuneata on the growth of E. coli

\begin{tabular}{|c|c|c|c|c|c|c|c|}
\hline $\begin{array}{c}\text { Exposure concentration }(\mu \mathrm{g} / \\
\mathrm{m}^{\mathrm{L})} *\end{array}$ & 10000 & 5000 & 2500 & 1250 & 500 & 250 & 125 \\
\hline $\begin{array}{c}\text { Growth inhibition percentage } \\
(\%)\end{array}$ & $100 \%$ & $100 \%$ & $100 \%$ & $96 \%$ & $80 \%$ & $72 \%$ & $38 \%$ \\
\hline
\end{tabular}

*Control group plate was regular $L B$ agar; Treated group plates were prepared by regular $L B$ agar with different concentrations of $H$. cordata and $S$. cuneata. 


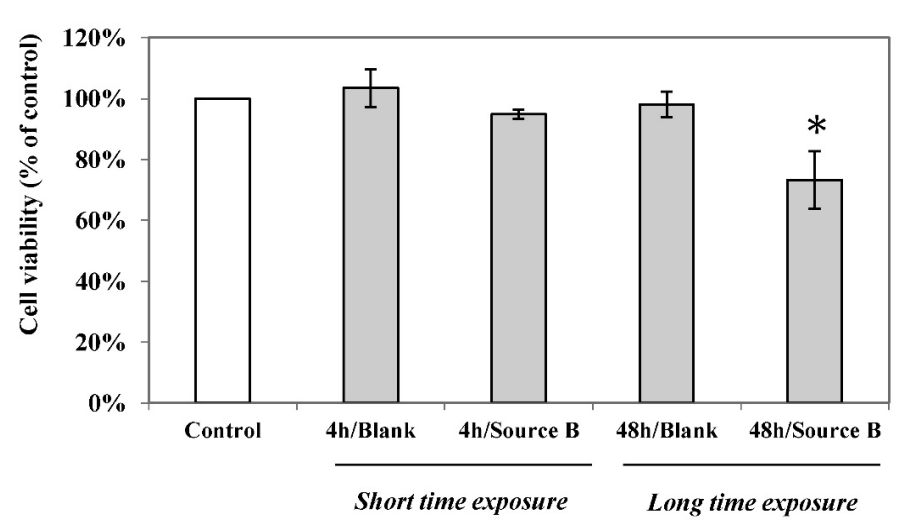

Figure 3. Cell viability of $E$. coli exposed to different time under the electronic transmission of source B. Values reported as percentages of the control group. Star indicating a value significantly different from the control group $(* \mathrm{p}<0.01)$

\section{Discussion}

The antibacterial effect of $H$. cordata and $S$. cuneata was well documented $[12,13]$, which are known to work in synergy and therefore often prescribed in pairs by Chinese Medicine practitioners. For this study, the mixed extract of these two herbs exhibited very effective inhibition to the growth of E. coli even at a low concentration (e.g. 1250 $\mathrm{ug} / \mathrm{ml}$ ). However, this is the first time that a nonlocal antibacterial effect of these two herbs was reported.

A number of studies reported that all chemicals have an intrinsic electromagnetic signature that carries the information and function of the molecule, and furthermore, the information and function can be separated from the molecules and transferred via physical fields [14-17]. In this study, the antibacterial properties of $H$. cordata and $S$. cuneata were successfully transferred to the E. coli cells, leading to significant growth suppression. The chemical makeup of these two herbs are very complex, including various compounds such as volatile oils, organic acids, flavonoids (quercetin, isoquercitrin, afzelin, hyperin, reyoutrin, rutin), and water-soluble polysaccharides [18], among which the flavonoids contain many ring structures therefore potentially functioning as "loop antenna" to facilitate the transferring of chemical information [6].

Water is believed to play a critical role in information transferring and storing $[16,17,19,20]$. For this work, source A, a diluted solution of $H$. cordata and $S$. cuneata, failed to yield a nonlocal effect, and while source B, the dried powder, achieved the inhibitory effect, which could be explained that 1 . water may interfere with the information transfer of the molecules and 2. a desired dosage is required for the success of information transferring. More work needs to be done to better understand the mechanism behind this matter.

Unlike the direct contact taking effect much faster, the nonlocal effect seemed to be an accumulative process as a four- hour exposure to the signal of the herbs did not affect the growth while a $48 \mathrm{~h}$ exposure caused significant growth reduction of $E$. coli. In addition, the potency of the transferred information seemed to be lower compared to the chemicals, which is comparable to the finding by Ebbers and Myle [6] that the information transfer of clotrimazole by scalar wave is about half as effective as the direct chemical drug application on the growth of yeast.

\section{Conclusion}

A nonlocal inhibitory effect of two Chinese Medicine herbs, $H$. cortata and S. cuneata, on the growth of $E$. coli was demonstrated by electronically transmitting the information of the extracted herbs. The "information" of the material was transferred without the mediation of water. On the other hand, the medicine dissolved in water as the source of information did not achieve information transfer, and the reasons behind this need to be further explored.

\section{Acknowledgment}

The authors would like to thank Dr. Yulong Wei for prescribing the $H$. cordata and S. cuneata for this study; we would also like to thank Yongdong Yang for assisting the installation of scalar wave experimental setup.

\section{Author contributions}

The authors Yu Chen and Zhong Zhen Cai contributed equally to this work; Methodology: Xuemei Bai, Yu Chen, Peng Gao and Zhong Zhen Cai; Data Curation: Yu Chen; Investigation: Yu Chen, Zhong Zhen Cai, Peng Gao and Qian Feng; Writing-Original Draft Preparation: Yu Chen, Xuemei Bai; Supervision: Xuemei Bai, Bruce Q. Tang.

\section{Funding}

The study was funded by ENN Group.

\section{References}

1. Ke YL, Chang FY, Chen MK, Li SL, Jang LS, et al. (2013) Influence of Electromagnetic Signal of Antibiotics Excited by Low-Frequency Pulsed Electromagnetic Fields on Growth of Escherichia coli. Cell Biochem Biophys 67: 1229-1237. [Crossref]

2. Oschman JL (2000) Energy Medicine: The Scientific Basis: Churchill Livingstone.

3. Alberto F, Mario L, Sara P, Settimio G, Antonella L, et al. (2014) Electromagnetic information delivery as a new tool in translational medicine. Int J Clin Exp Med 7: 2550-2556. [Crossref]

4. Heredia-Rojas JA, Villarreal-Treviño L, Rodríguez-De Fuente AO, Herrera Menchaca LI, Gomez-Flores R, et al. (2015) Antimicrobial effect of vancomycin electrotransferred water against methicillin-resistant Staphylococcus aureus variant. Afr $J$ Tradit Complement Altern Med 12: 104-108.

5. Rad I, Jalali K (2018) Electronic Transmission of Antibacterial Property Into Water at Extremely Low Frequency Range: A Preliminary Study. J Altern Complement Med 24: 431-438.

6. Ebbers JA, Meyl K (2014) Drug effects in yeast mediated by scalar waves. Med Sci 8: 58-62.

7. Pan Y, Sun DW, Han Z (2017) Applications of electromagnetic fields for nonthermal inactivation of microorganisms in foods: an overview. Trends Food Sci Technol 64 13-22.

8. Kreisl P (1998) Test on the transduction of acetic acid information via an electronic amplifier. Acta Medica Empirica 47: 17-24.

9. Thomas Y, Schiff M, Belkadi L, Jurgens P, Kahhak L, et al. (2000) Activation of human neutrophils by electronically transmitted phorbol-myristate acetate. Medical Hypotheses 54: 33-39.

10. Meyl K, Schnabl H (2015) Biological Signals Transmitted by Longitudinal Waves Influencing the Growth of Plants. Int J Environ Eng 8: 23-27.

11. Schnabl H, Meyl K (2018) Longitudinal magnetic waves trigger higher ATP-levels and extend the aging process of plants. Biol Eng Med 3:1-4.

12. Lai KC, Chiu YJ, Tang YJ, Lin KL, Chiang JH, et al. (2010) Houttuynia cordata Thunb Extract Inhibits Cell Growth and Induces Apoptosis in Human Primary Colorectal Cancer Cells. Anticancer Res 30: 3549-3556.

13. Junmin L, Zexin J, Hong S (2005) The Anti-Microbial Activity of the Extraction from Leaves of Sargentodoxa cuneata. J Chinese Med Mat 28: 906-909.

14. Foletti A, Ledda M, D’Emilia E, Grimaldi S, Lisi A, et al. (2012) Experimental finding on the electromagnetic information transfer of specific molecular signals mediated through the aqueous system on two human cellular models. J Altern Complement Med 18: $258-261$. 
15. Kim WH (2013) New Approach Controlling Cancer: Water Memory. J Vortex Sci Technol 1: 104.

16. Montagnier L, Aissa J, Del Giudice E, Lavallee C, Tedeschi A, et al. (2010) DNA waves and water. Quantitative Biol 306:1.

17. Montagnier L, Del Giudice E, Aïssa J, Lavallee C, Motschwiller S, et al. (2015) Transduction of DNA information through water and electromagnetic waves. Electromagnet Biol Med 34: 106-112.
18. Yang L, Jiang JG (2009) Bioactive components and functional properties of Hottuynia cordata and its applications. Pharmaceutical Biol 47:1154-1161.

19. Montagnier L, Aïssa J, Capolupo A, Craddock TJA, Kurian P, et al. (2017) Water Bridging Dynamics of Polymerase Chain Reaction in the Gauge Theory Paradigm of Quantum Fields. Water 9: 339.

20. Tang BQ, Li T, Bai X, Zhao M (2019) Rate limiting factors for DNA transduction induced by weak electromagnetic field. Electromagnet Biol Med 38: 55-65.

Copyright: (2019 Chen Y. This is an open-access article distributed under the terms of the Creative Commons Attribution License, which permits unrestricted use, distribution, and reproduction in any medium, provided the original author and source are credited. 